19 Revue d'histoire du XIXe siècle

Société d'histoire de la révolution de 1848 et des

révolutions du XIXe siècle

25 | 2002

Le temps et les historiens

\title{
Les Français face au temps de l'Empire
}

\section{Natalie Petiteau}

Údition électronique

DOI : $10.4000 /$ rh 19.417

ISSN : $1777-5329$

\section{Éditeur}

La Société de 1848

\section{Édition imprimée}

Date de publication : 1 décembre 2002

Pagination : 29-41

ISSN : 1265-1354

\section{Référence électronique}

Natalie Petiteau, «Les Français face au temps de l'Empire », Revue d'histoire du XIXe siècle [En ligne],

25 | 2002, mis en ligne le 07 mars 2008, consulté le 21 septembre 2021. URL : http://

journals.openedition.org/rh19/417 ; DOI : https://doi.org/10.4000/rh19.417

Ce document a été généré automatiquement le 21 septembre 2021.

Tous droits réservés 


\title{
Les Français face au temps de l'Empire
}

\author{
Natalie Petiteau
}

1 La cause semble désormais entendue : la période moderne s'achève, universitairement parlant, en 1799 et l'Empire ouvre donc l'ère contemporaine. Je laisse aux historiens de la Révolution le droit ou le devoir de défendre le rattachement des années 1789-1799 à l'ère contemporaine. Voilà qui renvoie à des débats dans lesquels, de toute façon, l'impact des années 1800-1815 est bien rarement pris en considération.

2 Pourtant, puisque l'Empire se trouve désormais ouvrir la période contemporaine, il devient d'autant plus essentiel de cesser d'en enfermer l'histoire dans les sphères au sein desquelles il a presque uniquement, jusqu'à présent, suscité l'intérêt. Il est vrai que plus que n'importe quelle période, l'Empire, notamment parce qu'il s'inscrit dans le temps très court de quinze années, a été le terrain privilégié d'une histoire essentiellement événementielle et même anecdotique, et dominée en tout cas, bien évidemment, par la reconstitution minutieuse de chacune des batailles de la période.

3 Or, on a abusivement négligé de jeter sur ces quinze années consulaires et impériales des regards neufs, porteurs des questionnements communs à l'ensemble d'une communauté historienne sans cesse en quête de renouvellements depuis les succès de l'école des Annales. C'est pourquoi j'ai personnellement souhaité organiser mes recherches postdoctorales dans le cadre d'une thématique que j'intitule "les Français et l'Empire». S'interroger sur l'attitude des Français à l'égard non seulement de ce régime politique, mais aussi des bouleversements humains induits par les mutations sociales qu'il a organisées, ou par les guerres dans lesquelles il a été impliqué, appelle donc, entre autres, une étude de la perception de ce moment historique par les contemporains, mais aussi par les historiens.

4 Finalement, comprendre les rapports que les Français ont à l'Empire, c'est analyser les lectures successives de ce temps qui signifie pour beaucoup de contemporains et d'historiens du XIX siècle densité des événements, mais aussi ouverture d'une ère nouvelle, alors même que nombre de mémorialistes témoignent de la perception d'un temps individuel souvent, mais pas forcément, marqué par un retour à un cours apaisé de 
l'existence. Manifestement, il existe différents «temps de l'Empire», selon les sources auxquelles on se réfère.

La lecture des correspondances entretenues par les contemporains de l'Empire ne révèle pas de réflexions générales sur les temps vécus par leurs auteurs ${ }^{1}:$ ces correspondances montrent bien au contraire des hommes et des femmes préoccupés du quotidien, sans jamais exprimer le sentiment de vivre des temps d'exception, du moins lorsqu'il s'agit de civils. Ce n'est qu'à l'heure de la mise en forme de leurs mémoires que quelques Français qui ont vécu l'Empire ont reconstitué a posteriori leur perception de ces temps.

6 Si l'expression « restauration » renvoie inévitablement l'historien aux années 1814-1815, il n'est pas certain que les hommes et les femmes du premier XIXe siècle n'aient pas aussi pensé aux années du Consulat et de l'Empire en terme de restauration. En tout cas, un témoignage de Chateaubriand prouve qu'il existe une volonté d'effacer en partie les temps révolutionnaires, si bien que 1800 serait entre autres vécu comme un temps de retour en arrière : en 1800, à Paris, il observe en effet que «sur les murailles étaient barbouillées ces inscriptions républicaines déjà vieillies : LIBERTE, EGALITE, FRATERNITE OU LA MORT. Quelquefois on avait essayé d'effacer le mot MORT, mais les lettres noires ou rouges reparaissaient sous une couche de chaux. Cette nation, qui semblait au moment de se dissoudre, recommençait un monde, comme ces peuples sortant de la nuit de la barbarie et de la destruction du Moyen Âge ${ }^{2}$. Notons que pour Chateaubriand, cette tentative d'effacer ce qui a été signifie en fait volonté de recommencement, de retour en arrière, après quoi il parle d'ailleurs des années du Consulat comme d'une époque de renaissance de l'ordre social ${ }^{3}$. Or, Charles de Rémusat a laissé un témoignage identique sur les réactions de ses concitoyens face à la recrudescence de cérémonial, aux premiers temps de l'Empire : on y voyait « les symptômes croissants de retour à l'ordre " 4 .

7 Pour d'autres mémorialistes, moins soucieux que Chateaubriand ou Rémusat de perspicacité dans la compréhension du temps écoulé, 1800 permet de renouer avec le cours normal de l'existence, de mettre entre parenthèses les années 1789-1799, autre tentative, donc, pour effacer le temps passé. Ainsi, pour la marquise de La Tour du Pin, la chute du Directoire est accueillie comme une heureuse nouvelle et le Consulat lui laisse avant tout l'agréable souvenir du retour des émigrés ${ }^{5}$ et de la restauration des plaisirs domestiques ${ }^{6}$. Victorine de Chastenay interprète elle aussi le moment de l'établissement du régime de Napoléon comme un temps de restauration parce qu'elle peut goûter le rétablissement progressif de son univers familier : les mémorialistes démontrent en fait que chacun n'envisage que le temps de son groupe social, sans pour autant oublier le temps strictement individuel des difficultés financières ou familiales qui ne cessent pas aussitôt que s'établit le nouveau pouvoir... ${ }^{7}$ Même la reine Hortense laisse entendre, dans ses mémoires, qu'elle a davantage vécu l'Empire au rythme de ses chagrins domestiques qu'à celui des grands événements politiques auxquels elle est pourtant directement mêlée ${ }^{8}$.

8 Quoi qu'il en soit, le sentiment de restauration n'est pas propre aux ci-devants nobles. Ainsi, le général Boulart conserve des années du Consulat le souvenir d'un temps de retour aux plaisirs, temps qu'il a goûté alors qu'il est en poste à Thionville : en évoquant la sociabilité et les plaisirs des soirées d'alors, il note qu'« il semblait qu'on eût à cœur de se dédommager du temps perdu » ${ }^{9}$. Et 1803 , alors qu'il est cette fois en poste à Besançon, chacun sentait que l'« on commençait à s'éloigner des temps désastreux de la Révolution ; la confiance s'était rétablie dans les relations sociales ; la prospérité renaissait, l'avenir souriait; on ne demandait pas mieux que de se dédommager pour le présent des 
privations du passé ; on s'entendait donc admirablement pour s'amuser ${ }^{10}$. Le Consulat semble donc être perçu comme un temps d'insouciance où chacun peut se laisser aller à vivre dans l'instant.

Or, les années de guerre, pour une raison inverse, encouragent bientôt également à cultiver l'art de vivre dans l'instant. À propos du temps de son mariage, en 1805, Boulart se souvient qu'«à cette époque, on était façonné à toutes les chances qui résultent de l'état de guerre; on réfléchissait beaucoup à l'avance, mais, une fois le parti pris, on se résignait à toutes les conséquences, et l'on profitait habilement pour le plaisir de tous les instants que le service de l'État ne réclamait pas impérieusement ${ }^{11}$. De cette ivresse à vivre dans l'instant, naît, Boulart le reconnait bien volontiers, l'habitude de dépenser sans compter, parce que «le présent était tout, et l'avenir paraissait si assuré qu'on ne s'en inquiétait pas; c'était un temps de délire et d'illusions " ${ }^{12}$. Le général Lasalle a lui aussi fort bien traduit comment les hommes de sa génération ont alors pu vivre sans penser au lendemain, en se satisfaisant de la plénitude d'un présent fait de gloire et d'aventures: " Moi, j'ai assez vécu à présent. Pourquoi veut-on vivre ? Pour se faire honneur, pour faire son chemin, sa fortune ; eh bien! j'ai 33 ans, je suis général de division. Savez-vous que l'empereur m'a donné l'année dernière 50000 livres de rentes ? c'est immense » et encore «[...] c'est déjà un plaisir assez grand que de faire la guerre ; on est dans le bruit, dans la fumée, dans le mouvement; et puis quand on s'est fait un nom, eh bien! on a joui du plaisir de se le faire ; quand on a fait sa fortune, on est sûr que sa femme, que ses enfants ne manqueront de rien : tout cela, c'est assez. Moi je puis mourir demain ${ }^{13}$.

Les contemporains de Napoléon semblent donc nombreux à avoir vécu les années de son règne dans la certitude d'avoir atteint une ère de fortune éternelle, au point de s'inscrire dans un temps immobile, sentiment exprimé même sous la plume d'un Alfred de Musset: «La mort elle-même était si belle alors, si grande, si magnifique dans sa pourpre fumante! Elle ressemblait si bien à l'espérance, elle fauchait de si verts épis qu'elle en était comme devenue jeune, et qu'on ne croyait plus à la vieillesse » ${ }^{14}$. Par ailleurs, Marguerite de La Tour du Pin, à propos de l'année 1813, rappelle que l'« on était si confiant dans la fortune de Napoléon, que l'idée ne venait à personne d'admettre qu'il eût d'autre ennemi à craindre que les frimas qui lui avaient été si fatals pendant la campagne de Russie ${ }^{15}$. Personne, donc, ne paraît envisager que l'avenir puisse ne pas ressembler au présent. Cependant, le sentiment d'immobilité peut naître de ce que ces années, si denses pour certains, ont été pour d'autres des années d'indifférence et de vacuité. La comtesse de Boigne note que sa vie a été d'une extrême monotonie durant les années de l'Empire : et parce qu'elle n'a que très peu pris part aux grands événements, elle n'a "guère de jalons pour fixer les époques " ${ }^{16}$.

11 Mais pour ceux qui ont subi les contrecoups des événements militaires, l'Empire a laissé le souvenir d'un temps de rupture. Encore enfant au temps des Cent Jours, la comtesse d'Agoult, dont le père décide de suivre Napoléon dans sa nouvelle campagne, tout en prenant la précaution de placer sa famille à l'étranger, a vécu l'année 1815 comme un tournant de son existence : «Et c'est ainsi que Napoléon Bonaparte, en venant soudain ressaisir la couronne impériale, en jetant par tout le monde le trouble et l'effroi, jetait du même coup, dans la paix de mon enfance, une première perturbation. Son épée conquérante, qui menaçait l'Europe, tranchait sans le savoir, dans l'ombre de mon existence, les premiers liens de l'habitude qui me rattachaient encore au berceau; elle me tirait brusquement de ce premier rêve doré du matin, commencé par l'enfant dans la nuit du sein maternel ${ }^{17}$. 
12 Mais aux Français qui ne perçoivent les malheurs des temps que lorsque leur vie personnelle est véritablement touchée, au paroxysme de 1815, s'ajoutent tous ceux que ces temps de guerre ont meurtri dans leur chair et leur âme. Pour eux bien sûr, l'Empire a pu laisser avant tout le souvenir d'un temps de désolation, loin de la légende d'un petit peuple qui aurait été unanimement nostalgique de la période napoléonienne. N'oublions pas, en effet, les paroles d'Alfred de Musset : « Jamais il n'y eut tant de nuits sans sommeil que du temps de cet homme ; jamais on ne vit se pencher sur les remparts des villes un tel peuple de mères désolées ; jamais il n'y eut un tel silence autour de ceux qui parlaient de mort ${ }^{18}$.

13 Aux témoignages qui rendent compte d'un temps individuel s'opposent ceux qui inscrivent les acteurs de l'épopée impériale dans un temps militaire et politique, perçu bien souvent comme un temps d'exception et de mutation.

Témoignant des conversations tenues dans les cercles proches de la cour consulaire, Charles de Rémusat donne de la société du temps de son enfance l'image d'un monde conscient de vivre un moment particulier de l'histoire, bruissant en permanence des hauts faits politiques et militaires d'un présent magnifié : «On n'imagine pas combien les faits et gestes du Consulat étaient l'entretien quotidien des familles, combien les souvenirs de la Révolution revenaient souvent dans la conversation, combien l'histoire contemporaine se faisait alors au grand jour ${ }^{19}$.

De même Boulart exprime clairement le sentiment d'avoir été le spectateur d'événements mémorables. Il en est ainsi de la cérémonie du sacre du 2 décembre 1804 : «je n'en ai pas moins été très satisfait et flatté d'avoir assisté, en qualité de témoin convoqué, à une cérémonie si grande, si bien ordonnée, si extraordinaire, et qui nous paraissait alors si pleine d'avenir. De nombreux siècles s'écouleront probablement avant que nos neveux ne voient quelque chose d'aussi prodigieux : on doit être fier d'avoir été acteur d'une pareille scène et s'enorgueillir de pouvoir en prendre acte ${ }^{20}$.

En dépit du froid sévère qui fait souffrir les hommes qui, comme Boulart, assistent à la cérémonie, celle-ci laisse le souvenir de l'apothéose « d'un être prodigieux » si bien que « le passé, le présent, l'avenir, absorbaient presque simultanément la pensée, et tenaient l'esprit dans une sorte de fascination $"{ }^{21}$. Si les témoins directs de la vie politique de ces années impériales ont l'impression, a posteriori, d'avoir vécu des années sans pareil, ils n'en ont pas moins, comme les mémorialistes témoignant de leur seule vie privée, tendance à recomposer le temps de l'Empire comme un temps immobile où présent, passé et avenir ne font plus qu'un. Toutefois, le présent apparaît à beaucoup de ces témoins directs des bouleversements politiques comme incomparable aux temps passés. À propos du sacre toujours, le comte de Lavalette parle de «la plus grande solennité qui ait été offerte depuis mille années au monde chrétien ${ }^{22}$. À l'autre extrémité de la période, le retour de l'île d'Elbe lui a laissé le souvenir d'« un événement immense, et tellement singulier, que les quatorze siècles de la monarchie n'avaient rien présenté de si extraordinaire ${ }^{23}$. Si bien que chaque témoin doit se sentir l'obligation de raconter ce temps d'exception ${ }^{24}$. L'Empire est en fait perçu comme «la grande époque » que chaque acteur est fier d'avoir vécue, au point de se complaire à vivre dans ce passé indéfiniment remémoré, comme pour s'enfermer, là encore, dans un temps immobile ${ }^{25}$. Quant aux batailles qui ponctuent ces temps de lutte entre la France et une partie de l'Europe, leurs acteurs, célèbres ou obscurs, en font des moments d'exception qui méritent seuls, finalement, de ponctuer la mémoire de ces années: Gourgaud, quelques jours après Austerlitz, écrit à sa famille en proclamant que l'on parlera longtemps de cette bataille ${ }^{26}$, 
Boulart qualifie Essling de «si longue, si éternelle journée » ${ }^{27}$, et le modeste François Imonet, caporal natif d'Avignon et blessé à Wagram, qualifie cette journée d'«immortelle " ${ }^{28}$. Étudier ce que ces années ont laissé dans les mémoires de leurs acteurs permet donc de saisir leur perception, à tout le moins leur recomposition, de ces temps rythmés par les combats.

Or, ce qui frappe précisément à la lecture de la majorité des mémorialistes de l'Empire, c'est la place accordée aux faits militaires : pour les officiers comme pour les combattants plus modestes, le temps de l'Empire est avant tout un temps ponctué par la guerre que chacun s'applique à reconstituer, restituant de ce fait une forme de temps individuel puisque chacun a eu sa propre vision des événements, au travers, toutefois, des sources officielles ${ }^{29}$. Ainsi les éphémérides du général Boyeldieu ne font état que des événements militaires ${ }^{30}$, et montrent en fait ce que pouvait être l'écho des Bulletins de la Grande Armée, où les hauts faits d'armes sont reconstitués heure par heure ${ }^{31}$. Et parce que chacun a en tête de telles éphémérides, on se résigne à la restauration de la monarchie grâce à une superposition mentale de plusieurs temps. Le capitaine Bertrand se rappelle comment l'Empereur restait alors leur Drapeau, leur point de ralliement: «Le souvenir de notre passé glorieux, précise-t-il, nous faisait oublier un moment les malheurs de la patrie, et nous sentions que notre cœur, avec toute notre âme, allait vers lui, bien que la discipline nous fit obéir au Drapeau blanc ${ }^{32}$.

Pourtant, ce temps, s'il est rythmé, dans la mémoire de chacun, par les grandes batailles, demeure bien souvent un temps imprécis. Auguste Thirion, par exemple, livre des souvenirs presque sans date où le temps est presque toujours évalué de façon imprécise ${ }^{33}$, à l'exception, toutefois, des passages consacrés à la campagne de Russie ${ }^{34}$, traces sans doute de ce qu'il aura conservé le célèbre $29^{e}$ bulletin, tandis qu'il indique plus systématiquement des dates ou des délais dès lors qu'il s'agit de sa vie familiale ${ }^{35}$. Il semble que moins la moisson de lauriers a été abondante, plus le militaire a négligé le temps collectif des combats pour s'inscrire de préférence dans le temps individuel de la vie affective. Toutefois, par l'engagement même dans une vie ponctuée de repères qui sont communs à un grand nombre de personnes, l'Empire a sans doute été un temps d'intériorisation du calendrier: désormais, on perçoit le temps en fonction de la grille précise des quantièmes et des mois. Or, en 1807 encore, un témoignage du baron Boulart révèle comment, parmi les troupes bivouaquant à proximité de Friedland, circule bientôt la nouvelle que le jour qui se lève est l'anniversaire de la bataille de Marengo ${ }^{36}$. Cet épisode montre en fait que les hommes connaissaient encore rarement la date du jour. Mais bientôt, tous les hommes déjà rentrés dans leurs foyers et pourvus d'une pension disposent d'un document reconstituant leurs états de service. Dès lors, ces pièces officielles ont constitué de précieux supports pour une acquisition, non seulement par les vétérans eux-mêmes, mais aussi par leurs familles, de la connaissance du calendrier de leur existence, normé de nouveau, depuis 1806, par le comput chrétien. Par le fait des formalités administratives induites par la retraite militaire, l'Empire signifie l'entrée dans une ère où le temps est perçu au travers de références universelles et non plus seulement en fonction de repères individuels. En cela aussi, l'Empire est bien le temps de l'entrée dans le contemporain.

Reste que ce temps est pour tous un temps fragmenté entre l'avant et l'après 1815 . Nombre de mémoires prouvent, par leur chronologie, que le temps qui mérite d'être conté s'arrête en 1815. Les raisons d'une telle démarche sont explicitées, par exemple, par le colonel Noël : « Me voilà à la fin de mes souvenirs militaires; car, quoique j’aie encore 
appartenu pendant quinze ans à l'armée, je n'ai presque rien à raconter de cette période de paix ${ }^{37}$. Or une telle attitude est également le fait des historiens qui proposent leur propre recomposition d'un temps collectif.

Les historiens du XIX ${ }^{\mathrm{e}}$ siècle ont toujours prétendu, mais bien souvent en vain, livrer une histoire objective de la période consulaire et impériale : en réalité, ils ont bien davantage exprimé leur opinion à propos de l'Empire, au point de le présenter tour à tour comme un temps soit condamnable soit mémorable ${ }^{38}$.

Si les contemporains de l'Empire n'ont pas forcément eu le sentiment de vivre des années sans pareil, les historiens ont quant à eux la vive conscience de se trouver face à une période exceptionnelle. Antoine-Clair Thibaudeau, notamment, l'exprime, en 1827, dans sa préface aux six volumes de son Histoire générale de Napoléon Bonaparte, en soulignant que les années 1789-1815 ont constitué certes un temps très court, mais dont l'influence sur le sort du monde a été immense, ouvrant de ce fait un vaste champ à l'histoire ${ }^{39}$. Si bien que la première préoccupation de tout historien est de souligner que le temps qui le sépare de son objet d'étude est une garantie d'objectivité. Dès lors, chaque œuvre majeure consacrée, au XIX ${ }^{e}$ siècle, à l'histoire des années 1800-1815, débute par une profession de foi où l'on sent combien l'auteur est soucieux de se montrer comme débarrassé du poids de ce passé et donc apte à en livrer une histoire objective. Ainsi, en 1822, Arnault ouvre sa Vie politique et militaire de Napoléon par cette affirmation : « Napoléon n'est plus. Le temps où l'on peut écrire son histoire est arrivé $"{ }^{40}$. Pourtant, en 1827 , Thibaudeau ne reconnaît à aucun de ses prédécesseurs l'aptitude à l'objectivité : "Quel observateur était assez clairvoyant pour saisir la vérité à travers les nuages dont [cette histoire] était enveloppée, quel acteur assez impartial pour la révéler, quel témoin assez courageux pour la dire ? " ${ }^{41}$

Les lendemains de 1830 donnent un nouvel élan à l'historiographie napoléonienne, à l'heure où des auteurs comme Tissot viennent condamner implicitement les prétentions de leurs prédécesseurs : selon lui, « dans le cours des années qui ont suivi 1814, l'annonce d'une histoire de Napoléon aurait équivalu à celle d'une entreprise de louanges outrées ou d'invectives sanglantes. Aujourd'hui que les passions refroidies se sont fondues dans un besoin de justice et d'impartialité, et que [1]es fautes [de Napoléon] ne sont pas plus contestées que son génie, une telle publication ne saurait soulever de défiance ${ }^{42}$. Et si cet auteur d'une nouvelle Vie de Napoléon se croit autorisé à se proclamer le premier historien enfin objectif, c'est parce que le temps écoulé a permis l'accumulation de mémoires auxquels puiser et l'accès à des sources jusqu'alors inconnues. Et si Louis Ardant revendique lui aussi, pour son Histoire de Napoléon, le droit à l'objectivité, c'est parce que l'avènement d'un roi-citoyen et le retour du drapeau tricolore laissent espérer, selon lui, que les haines exprimées sur la tombe de Sainte-Hélène sont définitivement éteintes ${ }^{43}$.

Pourtant, en 1900, lord Rosebery ne semble pas avoir encore trouvé une seule histoire satisfaisante de la vie de Napoléon : «Qui nous écrira la vie de Napoléon comme elle doit être écrite? Jusqu'ici, poser la question eût été peine perdue. Les préjugés et les passions du temps étaient encore trop près de nous pour qu'on pût songer à écrire un tel livre. Et aujourd'hui même nous n'en sommes pas bien éloignés, car la reine Victoria avait déjà deux ans à l'époque de la mort de Napoléon et il existe probablement encore des personnes qui l'ont vu. Puis le Second Empire a ranimé, multiplié ces sentiments presque dans leur intensité première et la réaction qui a suivi le Second Empire en a prolongé 
encore l'existence. Peut-être ne sommes-nous pas assez complètement sortis de la sphère historique de Napoléon pour qu'il soit possible d'écrire sa vie ${ }^{44}$.

Ainsi, pour paraphraser Conan Doyle, la Grande Ombre de Napoléon s'étendrait sur tout le XIX' siècle, au point qu'en 1900 les Européens vivraient en un temps encore marqué par l'emprise napoléonienne. Le siècle entier n'aurait donc été qu'un prolongement des temps impériaux, empêchant par là même les historiens de livrer des œuvres objectives ${ }^{45}$ . Du reste, la revendication des auteurs demeure celle d'une histoire de Napoléon et non d'une histoire de la période. Napoléon a de toute façon capté systématiquement les regards des historiens qui, dans un souci d'objectivité, mais aussi dans la logique de l'historiographie du XIX siècle, ont inlassablement reconstitué les événements de la vie de l'empereur, respectant une matrice qui met notamment l'accent sur l'enfance de leur héros: celle-ci contiendrait en germe le destin futur de l'empereur. L'ouvrage de Touchard-Lafosse et Saint-Amant, publié en 1825, en est un témoignage: «La nature semble hâter pour lui cette saison de la vie. Étranger aux jeux de ses camarades, il se montre silencieux, réservé, méditatif. Son premier âge est empreint d'une maturité inaccoutumée [...]. Son imagination exaltée porte son existence tout entière dans l'avenir ; les temps futurs semblent s'offrir à lui sous l'aspect qu'ils auront : on dirait qu'il pressent l'élévation que lui prépare le destin $"{ }^{46}$.

Finalement, les historiens du XIX ${ }^{e}$ siècle ont enfermé l'histoire de l'Empire dans un temps uniquement rythmé par les événements de la vie militaire et diplomatique ${ }^{47}$, se contentant donc de livrer une chronique, au sens littéral, se prononçant par ailleurs sur l'opportunité de condamner ou de louer ces temps bouleversés par les combats ${ }^{48}$.

Dans les œuvres consacrées aux années 1800-1815, les historiens du XIX ${ }^{e}$ siècle ont en fait avant tout traduit leurs opinions politiques et révélé comment chaque période a repensé les temps napoléoniens. On sait combien les années 1814-1816 ont vu se multiplier les pamphlets fortement hostiles à Napoléon ${ }^{49}$, tandis que, dès les années 1820 , se développe une légende dorée qui trouve son apogée sous la Monarchie de Juillet après quoi, et Rosebery l'a bien remarqué, le Second Empire a donné un nouvel essor à la légende noire. Le présent de chaque historien a donc été déterminant dans l'interprétation du temps de l'Empire ${ }^{50}$.

27 Nombreux sont les historiens qui ont contribué à la construction de la légende dorée, faisant de Napoléon un acteur de l'histoire à nul autre pareil, placé sur un piédestal pour son génie politique, pour son aptitude à réorganiser la France ou pour son habileté militaire, donnant à son règne le caractère d'un temps mémorable, caractère que, selon Arnault, même les contemporains auraient perçu par comparaison entre le passé et le présent: «Comparant le présent au passé, la nation bénissait sincèrement l'homme auquel elle était redevable d'une prospérité qui semblait ne pouvoir qu'augmenter ${ }^{51}$.

De même, pour Louis Ardant, «Napoléon remplit le monde de son nom et couvrit les armes françaises d'une gloire impérissable. Grand capitaine, adroit politique, profond législateur, il surpassa les plus hautes célébrités de l'histoire ${ }^{52}$. Ou encore, pour Tissot, «il est des hommes privilégiés sur qui la postérité prononce un jugement définitif sans qu'il ait passé par l'épreuve du temps, ou plutôt à l'égard desquelles elle ne fera que ratifier la conviction unanime d'une génération encore palpitante des souvenirs de leur vie, et dont la voix aura parlé trop haut pour n'être pas entendue. Napoléon est un exemple de cette vérité, lui dont la grande figure historique s'est reflétée avec tout son éclat dans la fin d'un siècle et dans le commencement d'un autre; homme étonnant, et qui grandit à être vu à distance $»^{53}$. 

proclame bien au contraire que l'éloignement dans le temps ne peut que renforcer le prestige de Napoléon. Pour Norvins également, «dans l'espace de plusieurs siècles, l'histoire ne présente pas un homme à qui Napoléon puisse être comparé. [...] Dans cent ans, on ne comprendra ni l'apparition ni la destruction de cet homme à part dans l'histoire comme dans la nature, qui, d'une île de la Méditerranée, s'élevant tout à coup sur l'Europe, la domina pendant vingt ans, disparut de la terre, et laissa ses débris au milieu des flots ${ }^{54}$. Mais à se laisser impressionner par le caractère exceptionnel du souverain dont ils tentent de connaître le règne, les historiens du XIX ${ }^{\mathrm{e}}$ siècle ont quelque peu négligé de mettre cette période en perspective. Elle est plus souvent lue comme moment d'exception que comme un temps véritablement fondateur. On loue l'œuvre législative et administrative sans toujours la mettre en rapport avec les temps présents. Toutefois Thiers, dans son ultime volume de l'Histoire du Consulat et de l'Empire, paru en 1862, a rendu hommage "au père de l'administration moderne ", et "au plus grand organisateur qui ait paru dans le monde ${ }^{55}$. Du reste, parce que Napoléon a agi en différents domaines, et parce qu'il n'a pas la même attitude à l'égard de la nation selon qu'on le considère en 1799, en 1807, en 1814 ou en 1815, le temps du Consulat et de l'Empire est fractionné par les historiens: on se souvient bien sûr de Larousse qui considère que l'homme politique qu'était Bonaparte est mort à Saint-Cloud, le 19 brumaire. Mais n'oublions pas que, dès le premier XIX ${ }^{\mathrm{e}}$ siècle, certains historiens voient en 1807, année d'engagement dans la guerre d'Espagne, un moment de rupture entre l'Empire mémorable et l'Empire condamnable ${ }^{56}$. de la France contemporaine que certains auteurs qui opèrent ce travail de mise en perspective voient dans l'Empire un temps à jamais condamnable. Taine en est un remarquable exemple. S'il reconnait bien volontiers que «[Napoléon] a fait la France moderne ${ }^{57}$, c'est parce qu'il a non seulement établi un pouvoir exécutif concentré et fort mais aussi parce qu'il a réduit au silence l'opinion publique et établi l'isolement de chaque individu ${ }^{58}$ : la France napoléonienne connaît de ce fait un contraste frappant avec la France d'avant 1789 et c'est précisément parce que le règne de Napoléon est l'avènement d'une nouvelle organisation sociale que ce temps est, aux yeux de Taine, absolument condamnable ${ }^{59}$.

31 Pour Michelet en revanche, c'est à l'aune de l'œuvre du Second Empire qu'il faut prononcer son jugement sur les années 1800-1815. Il insiste sur le parallèle à établir entre le César d'Austerlitz et celui de Sedan ${ }^{60}$. L'Empire est avant tout le temps d'un nombre de morts effroyable ${ }^{61}$. Et si cette période est bien à ses yeux fondatrice du contemporain, c'est parce qu'elle inaugure tout ce que le XIX siècle présente de regrettable pour l'histoire humaine : « ceux qui croient que le passé contient l'avenir, et que l'histoire est un fleuve qui s'en va identique, roulant les mêmes eaux, doivent réfléchir ici et voir que très souvent un siècle est opposé au siècle précédent, et lui donne parfois un âpre démenti. Autant le dix-huitième siècle, à la mort de Louis XIV, s'avança légèrement sur l'aile de l'idée et de l'activité individuelle, autant notre siècle, par ses grandes machines (l'usine et la caserne) attelant les masses à l'aveugle, a progressé dans la fatalité " ${ }^{62}$.

Pour Michelet en effet, vers 1800, partout en Europe, le phénomène majeur est l'enrégimentation, dans l'usine ou dans la caserne ${ }^{63}$, mais surtout il déplore que «l'esprit de guerre avait alors gagné le monde " ${ }^{64}$. L'Empire est donc là encore un temps condamnable, parce qu'il a inauguré pour les hommes du nouveau siècle les maux dont ils 
ont depuis trop souvent souffert: parce que la logique du temps de guerre relie Sedan à Austerlitz, le XIX siècle a été lourdement marqué par l'entreprise napoléonienne. Pour Michelet comme pour Taine, qui sont parmi les premiers à inscrire l'Empire dans un temps séculaire, le XIX ${ }^{e}$ siècle est le siècle de Napoléon, mais ils le proclament pour mieux le déplorer.

Manifestement, le temps des historiens de l'Empire, si, du moins, on se limite à interroger l'historiographie du XIXe siècle, n'est que partiellement celui des contemporains. Ils ont oublié que, pour beaucoup de ces derniers, le temps présent n'était pas vécu comme un temps linéaire mais plutôt réversible, pour reprendre l'expression de Krzysztof Pomian ${ }^{65}$. Finalement, les Français qui ont vécu sous le règne de Napoléon n'ont-ils pas fort bien écouté ce qu'a proclamé le nouveau chef de la France au lendemain de brumaire : «La révolution est finie, elle est fixée aux principes qui l'ont commencée ». Bonaparte a donc lui-même invité ses concitoyens à vivre ces années 1800-1815 comme un retour en arrière. Mais, à l'heure de la remémoration, certains mémorialistes ont aussi montré la densité de ce temps rythmé par ce qu'ils ont jugé comme les heures glorieuses du règne. Si bien que les historiens les ont suivis sur la voie de la reconstruction de la densité événementielle pour donner finalement du temps de l'Empire l'idée d'un temps linéaire : en obéissant aux règles qui veulent alors que seule la reconstitution chronologique des événements garantisse l'objectivité de l'histoire, ils ont privé pour longtemps les Français d'une inscription de l'Empire dans un étagement de temporalités. On sait en effet que plus que pour d'autres périodes, l'histoire économique et sociale de l'Empire a été largement négligée. Or, que signifiait pour tel paysan ce temps de consolidation de la propriété des biens nationaux, de confirmation de la disparition des droits seigneuriaux mais aussi, bien souvent, de longue absence d'un fils enrôlé dans l'armée?

Or, ce temps de l'Empire demeure un temps défini par les élites, qu'ils s'agisse des mémorialistes ou des historiens: en dehors des rares traces laissées par les anonymes dans les dossiers militaires, comment savoir la perception du temps de l'Empire par l'ensemble des contemporains, par les petits, les obscurs, les sans-grade? La nature des sources offertes à nos investigations nous empêchera donc toujours de traiter dans toutes ses dimensions cette belle question de l'écart entre temps des contemporains et temps de l'historien...

\section{NOTES}

1. Voir par exemple la correspondance d'Annette de Mackau ou celle de général Bertrand, pour ce qui est des correspondances publiées ; Correspondance d'Annette de Mackau, comtesse de Saint-Alphonse, dame du Palais de l'impératrice Joséphine (1790-1870), éditée par Chantal de Toutier-Bonazzi, Paris, SEVPEN, 1967, 459 p. ; général Bertrand, Lettres à Fanny, 
1808-1815, annotées et présentées par Suzanne de la Vaissière-Orfila, Paris, Éditions Albin Michel, 1979, 500 p.

2. François-René de CHATEAUBRIAND, Mémoires d'outre-tombe, Bibliothèque de La Pléiade, Paris, Éditions Gallimard, 1951, volume 1, p. 437.

3. Idem, p. 510.

4. Charles de RÉMUSAT, Mémoires de ma vie. tome 1 : Enfance et jeunesse. La Restauration libérale (1797-1820), présentés et annotés par Charles H. Pouthas, Paris, Librairie Plon, 1958, p. 52.

5. Marguerite de LA TOUR du PIN, Mémoires (1778-1815) suivis d'extraits inédits de sa correspondance (1815-1846), avec une introduction et des notes de Christian de LiedekerkeBeaufort, collection « Le Temps retrouvé », Paris, Éditions du Mercure de France, 1989, p. 272 notamment.

6. Idem, p. 287.

7. Victorine de CHASTENAY, Mémoires, 1771-1815, collection « L'histoire en mémoires », Paris, Librairie Académique Perrin, 1987, p. 293.

8. Voir notamment son témoignage lors de la proclamation de l'Empire : Mémoires de la reine Hortense, publiés par le prince Napoléon, Paris, Librairie Plon, 1927, tome 1, p. 166.

9. Mémoires du général d'artillerie baron Boulart, présentés par Jacques Jourquin, Bibliothèque napoléonienne, Paris, Éditions Tallandier, 1992, p. 113.

10. Idem, p. 120.

11. Idem, p. 132.

12. Idem, p. 213.

13. François-Guy HOURTOULLE, Le Général comte Lasalle, Paris, 1979, cité par Abel POITRINEAU, « Fonctionnarisme militaire ou catharsis guerrière ? » dans Paul VIALLANEIX et Jean EHRARD [dir.], La bataille, l'armée, la gloire, 1745-1871, ClermontFerrand, Publications de la faculté des lettres et sciences humaines de Clermont-Ferrand, 1985, tome 1, p. 216.

14. Alfred de MUSSET, La Confession d'un enfant du siècle, Folio, Paris, Éditions Gallimard, 1973 ( $1^{\text {ère }}$ édition 1836), pp. 20-21.

15. Marguerite de LA TOUR du PIN, Mémoires..., ouv. cité, p. 339.

16. Mémoires de la comtesse de Boigne, née d'Osmond, édition présentée et annotée par JeanClaude Berchet, Paris, Éditions du Mercure de France, 1982, volume 1, p. 169.

17. Mémoires, souvenirs et journaux de la comtesse d'Agoult, présentation et notes de Charles F. Dupêchez, Paris, Éditions du Mercure de France, 1990, tome 1, p. 64.

18. Alfred de MUSSET, La confession d'un enfant du siècle, ouv. cité, pp. 20-21.

19. Charles de RÉMUSAT, Mémoires de ma vie, ouv. cité, p. 33.

20. Mémoires du général d'artillerie baron Boulart, ouv. cité, pp. 124-125.

21. Idem, pp. 125-126.

22. Mémoires et souvenirs du comte de Lavalette (1769-1830), édition présentée et annotée par Stéphane Giocanti, Paris, Éditions du Mercure de France, 1994, p. 252.

23. Idem, p. 332.

24. Idem, p. 25.

25. Capitaine DESBCEUFS, Les étapes d'un soldat de l'empire. Souvenirs (1800-1815), Paris, Librairie des Deux Empires, 2000 (1 1 ère édition en 1901), p. IX.

26. Archives nationales, 314 AP 9, fonds Gourgaud, lettre du général Gourgaud à sa famille, 24 frimaire an XIV.

27. Mémoires du général d'artillerie baron Boulart, ouv. cité, p. 218. 
28. Service historique de l'armée de terre, dossier de pension de François Imonet, 2 Yf 45106.

29. De ces mémoires essentiellement occupés du fait militaire témoignent notamment les Mémoires du général baron de Marbot, édition présentée et annotée par Jacques Garnier, Paris, Éditions du Mercure de France, 1983, 2 volumes.

30. Général BOYELDIEU et général TESTE, Souvenirs de deux généraux du Premier Empire, Paris, Éditions Teissedre, 1999, p. 44 notamment.

31. Napoléon BONAPARTE, Proclamations, ordres du jour, bulletins de la Grande Armée, collection 10-18, Paris, 1964, $184 \mathrm{p}$.

32. Capitaine BERTRAND, Mémoires. Grande Armée, 1805-1815, Paris, Librairie des Deux Empires, 1999, p. 154.

33. Auguste THIRION, Souvenirs militaires, Paris, Librairie des Deux Empires, 1998, $197 \mathrm{p}$.

34. Idem, pp. 92-93.

35. Idem, p. 56 et p. 59 par exemple.

36. Mémoires du général baron de Marbot, ouv. cité, p. 166.

37. Colonel NOËL, Souvenir militaire d'un officier du Premier Empire (1795-1832), Paris, Librairie des Deux Empires, 1999, p. 199.

38. Pour de plus longs développements sur ce thème, je me permets de renvoyer à Natalie PETITEAU, Napoléon, de la mythologie à l'histoire, L'Univers historique, Paris, Éditions du Seuil, 1999, $439 \mathrm{p}$.

39. Antoine-Clair THIBAUDEAU, Histoire générale de Napoléon Bonaparte, de sa vie privée et de sa vie publique, de sa carrière politique et militaire, de son administration et de son gouvernement, Paris, Éditions Ponthieu, 1827, 6 volumes, préface de l'auteur non paginée.

40. A. V. ARNAULT, Vie politique et militaire de Napoléon, Paris, Éditions Émile Babeuf, 1822, introduction non paginée.

41. Antoine Clair THIBAUDEAU, Histoire générale de Napoléon Bonaparte, ouv. cité, préface de l'auteur non paginée.

42. Pierre-François TISSOT, Histoire de Napoléon rédigée d'après les papiers d'État, les documents officiels, les mémoires et les notes secrètes de ses contemporains suivie d'un précis sur la famille Bonaparte et précédée de réflexions générales sur Napoléon, Paris, Éditions DelangeTaffin, 1833, 2 volumes, 468 et $488 \mathrm{p}$, prospectus joint.

43. Louis ARDANT, Histoire de Napoléon. Détails sur sa famille, sa naissance, son éducation, son mariage, ses conquêtes, ses généraux, son exil et sa mort, Paris, Éditions Didier, 1833, p. 5.

44. Lord ROSEBERY, Napoléon. La dernière phase, traduit par Augustin FILON, Paris, Éditions Hachette, 1901 (1 ${ }^{\text {ère }}$ édition 1900), p. 1.

45. Ce qui renvoie à l'affirmation de l'historien Thiénot : «L'histoire ne naît pour une époque que quand elle est morte tout entière ", cité par Jean LEDUC, Les Historiens et le temps, Points-Histoire, Paris, Le Seuil, 1999, p. 60.

46. Georges TOUCHARD-LAFOSSE, J.-S. SAINT-AMANT, Précis de l'histoire de Napoléon, du Consulat et de l'Empire avec les réflexions de Napoléon lui-même sur les événements et les personnages les plus importants de son époque suivi d'un examen politique et littéraire des ouvrages qui se rattachent le plus immédiatement à l'histoire de Napoléon, Paris, Éditions A. Thoisnier-Desplaces, 1825, p. 9.

47. Voir notamment baron BIGNON, Histoire de France depuis le 18 brumaire (novembre 1799) jusqu'à la paix de Tilsitt (juillet 1807), Paris, Éditions Béchet/Éditions Firmin Didot, 1829-1850, 14 volumes ; F. TISSOT, Histoire de Napoléon, ouv. cité, ou Louis ARDANT, Histoire de Napoléon, ouv. cité. 
48. Thibaudeau est l'un des rares à mettre l'accent sur les aspects civils de l'histoire de la période.

49. Jean TULARD, L'Anti-Napoléon. La légende noire de l'empereur, Archives, Paris, Éditions Gallimard, 1965, $260 \mathrm{p}$.

50. Je renvoie pour de plus amples développements à Natalie PETITEAU, Napoléon, de la mythologie à l'histoire, ouv. cité.

51. A. V. ARNAULT, Vie politique et militaire de Napoléon, ouv. cité, volume 1, p. 124.

52. Louis ARDANT, Histoire de Napoléon, ouv. cité, p. 5.

53. Pierre-François TISSOT, Histoire de Napoléon..., ouv. cité, prospectus.

54. Jacques MARQUET de MONTBRETON de NORVINS, Histoire de Napoléon, $4^{e}$ édition, Paris, Éditions Furne, 1833 (1 ${ }^{1}$ è édition 1827-1828), volume 1, pp. VII-VIII.

55. Adolphe THIERS, Histoire du Consulat et de l'Empire, Paris, Éditions Paulin-Lheureux, 1862 , tome 20, p. 726 et p. 730.

56. Voir par exemple Georges TOUCHARD-LAFOSSE et J.-S. SAINT-AMANT, Précis de l'histoire de Napoléon, ouv. cité, p. 136.

57. Hippolyte TAINE, Les Origines de la France contemporaine. Tome 2 : La Révolution et le régime moderne, Collection Bouquins, Paris, Éditions Laffont, 1986 (1 1 ère édition 1890-1893), p. 372.

58. Idem, p. 461.

59. Idem, p. 464.

60. Jules MICHELET, Histoire du XIX siècle. Directoire. Origine des Bonaparte, Paris, Librairie Germer Baillière, 1872, p. VII.

61. Idem, p. VIII.

62. Idem, p. IX.

63. Idem, pp. X-XI.

64. Idem, p. XXII.

65. Krzysztof POMIAN, L'Ordre du temps, Paris, Éditions Gallimard, Bibliothèque des histoires, 1984, p. 56.

\section{RÉSUMÉS}

Il existe différentes perceptions du temps de l'Empire. Les mémorialistes civils ont le sentiment d'un retour à un temps d'apaisement et de restauration d'un cours habituel de l'existence, tandis que les militaires disent combien les temps de l'Empire sont avant tout caractérisés par la densité des événements guerriers et constituent des temps d'exception. Avec les historiens, dès les lendemains de 1815, les années 1800-1815 sont érigées en temps mémorables. Pour l'heure cependant, on ne connaît guère que le temps des élites.

The French and the time of the Empire. There were various perceptions of the time of the Empire. The civil memorialists had the feeling of a return to a time of appeasement and restoration of a usual course of the existence. The soldiers said how much the times of the Empire were first and foremost characterised by the density of the warlike events and how they constitute times of exception. After 1815, with historians, the years 1800-1815 were set up in memorable times. However, for now, one hardly knows but the time of the elite. 
AUTEUR

NATALIE PETITEAU

Maître de conférences à l'Université d'Avignon 